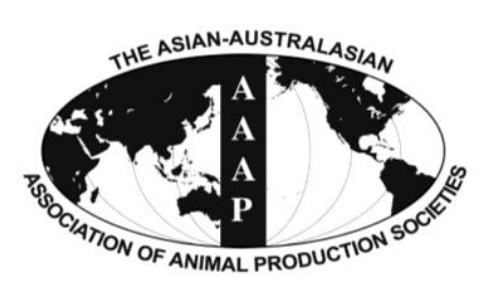

\title{
Genome-wide Association Study for Warner-Bratzler Shear Force and Sensory Traits in Hanwoo (Korean Cattle)
}

\author{
C. G. Dang ${ }^{\text {a }}$, S. H. Cho ${ }^{1, a}$, A. Sharma, H. C. Kim, G. J. Jeon, S. H. Yeon, S. K. Hong ${ }^{2}$, \\ B. Y. Park ${ }^{1}$, H. S. Kang, and S. H. Lee* \\ Hanwoo Experiment Station, National Institute of Animal Science, Rural Development Administration, \\ Pyeongchang 232-952, Korea
}

\begin{abstract}
Significant SNPs associated with Warner-Bratzler (WB) shear force and sensory traits were confirmed for Hanwoo beef (Korean cattle). A Bonferroni-corrected genome-wide significant association $\left(\mathrm{p}<1.3 \times 10^{-6}\right)$ was detected with only one single nucleotide polymorphism (SNP) on chromosome 5 for WB shear force. A slightly higher number of SNPs was significantly ( $<<0.001)$ associated with WB shear force than with other sensory traits. Further, 50, 25, 29, and 34 SNPs were significantly associated with WB shear force, tenderness, juiciness, and flavor likeness, respectively. The SNPs between $p=0.001$ and $p=0.0001$ thresholds explained $3 \%$ to $9 \%$ of the phenotypic variance, while the most significant SNPs accounted for $7 \%$ to $12 \%$ of the phenotypic variance. In conclusion, because WB shear force and sensory evaluation were moderately affected by a few loci and minimally affected by other loci, further studies are required by using a large sample size and high marker density. (Key Words: Genome-wide Association Study, Warner-Bratzler Shear Force, Sensory Traits, Hanwoo Beef)
\end{abstract}

\section{INTRODUCTION}

As a consequence of the recent economic growth, the human diet has dramatically changed, with an increasing demand for healthier meat. The eating quality of beef is the result of a combination of factors, including tenderness, juiciness, and flavor likeness (Koohmararaie et al., 2002), which contribute to the consumer's perception of meat palatability or satisfaction (Jeremiah, 1982). The tenderness is usually considered the most important quality aspect of meat and criterion of acceptability (Love, 1994;

\footnotetext{
* Corresponding Author: Seung Hwan Lee. Tel: +82-33-3300717, Fax: +82-33-330-0660, E-mail: slee46@korea.kr

${ }^{1}$ Animal Product Research and Development Division, National Institute of Animal Science, Rural Development Administration, Suwon 441-706, Korea.

${ }^{2}$ National Institute of Animal Science, Rural Development Administration, Suwon 441-706, Korea.

${ }^{a}$ Chang Gwon Dang and Soo Hyun Cho contributed equally to this work.

Submitted Oct. 29, 2013; Revised Mar. 2, 2014; Accepted Apr. 11, 2014
}

Koohmaraie et al., 1994). Also, a positive correlation between Warner-Bratzler (WB) shear force values and the sensory assessment of beef tenderness was investigated (Caine et al., 2003; Destefanis et al., 2008). Beef flavor intensity is an important component of meat palatability, and genetic factors are known to influence these traits (Shahidi and Rubin, 1986). Previous work has focused on flavor desirability and its relation to sensory tenderness and juiciness among 12 muscles from grain-fed beef (Carmack et al., 1995). Meat quality is an important factor in the beef industry because the consumer is willing to pay more for higher quality meat products. In particular, consumers have shown a strong willingness to pay a premium for "tender meat" that combines superior tenderness with leanness (Shackelfold et al., 2001; Shackelford et al., 2005).

Beef quality is evaluated by consumers based on sensory considerations of tenderness, flavor likeness, and juiciness (Cho et al., 2010). Traditional genetic improvement programs have focused on quantitative genetic theories using statistical analyses of phenotypes and pedigree information to determine animals with genetic 
merit. However, the palatable quality of beef is usually measured post-slaughter, which means it is hard to measure and has low heritability (Marshall, 1999). Thus, classical selection based on records is not a feasible option.

Given these conditions, genome-assisted selection, whereby DNA information linked to quantitative trait loci (QTL) of palatable quality traits are used, has the potential to improve the selection of low heritability sensory traits. Several DNA markers associated with meat quality traits have been identified in the last decade. In cattle, for meat and carcass quality, 2 genes account for moderate genetic effects on meat tenderness, including calpain 1 (CAPNI) and calpastatin (CAST), which have been confirmed in previous studies (Page et al., 2002; Page et al., 2004; Casas et al., 2005; White et al., 2005; Casas et al., 2006; Schenkel et al., 2006).

In order to achieve more targeted meat production in the Hanwoo beef industry, the incorporation of DNA marker information into genetic selection methods, such as markerassisted selection, to identify animals destined for feedlots or seed stocks could be a useful management tool (Rolf et al., 2010). Identifying causal genes affecting quantitative traits may have the potential to improve the efficiency of animal selection and elucidate the underlying, biological mechanisms of quantitative traits.

Recently, the genome-wide association study (GWAS) has been used to identify the causal genes of important genetic traits in livestock. The GWAS uses sequence variations in the whole genome in combination with phenotypic and pedigree information to perform an association analysis and identify the significant genes or regulatory elements for the selection of particular traits. This technique can be applied to control the evolution of a significant gene associated with palatable beef. Therefore, the aim of this study was to identify the significant single nucleotide polymorphism (SNPs) associated with WB shear force and sensory traits using GWAS methodology and evaluate the performance of these traits for the prediction of a phenotype in Hanwoo (Korean cattle).

\section{MATERIALS AND METHODS}

\section{Animals}

A total of 288 cattle were genotyped, including 116 cows, 90 bulls, and 82 steers. The samples were obtained from the Hanwoo experimental station in Pyeongchang, Gangwon Province, Korea. The data were comprised of paternal half-sib pedigrees from 62 sires.

\section{Warner-Bratzler shear force}

The WB-shear force of cooked steaks was measured according to the method described by Wheeler et al. (2000). At $48 \mathrm{~h}$ postmortem, $2.5-\mathrm{cm}$ thick meat steaks weighing $\sim 80 \mathrm{~g}$ were put into polyethylene bags. The bags were heated in a water bath at $80^{\circ} \mathrm{C}$ for $30 \mathrm{~min}$ until the internal temperature of the steak reached $70^{\circ} \mathrm{C}$. After cooking, the samples were cooled at room temperature for $30 \mathrm{~min}$. Six to eight representative, $1.27-\mathrm{cm}$ diameter cores were removed from each steak parallel to the muscle fiber. Shear force values were determined with a Warner-Bratzler shear force attachment on an Instron Universal Testing machine (Instron Corporation, Canton, MA, USA) with the following operating parameters: load cell, $50 \mathrm{~kg}$ and crosshead speed, $200 \mathrm{~mm} / \mathrm{min}$. Each core sample was sheared once across the center of the core perpendicular to the muscle fiber. The WB shear force value was the mean of the maximum forces required to shear each set of core samples.

\section{Sensory evaluation}

Longssimus dorsi (LD) muscles were obtained at $48 \mathrm{~h}$ postmortem, vacuum packaged, and aged at $2^{\circ} \mathrm{C}$ for $7 \mathrm{~d}$. To prepare beef strips, the meat blocks were cut into $75 \times 20 \times 4$ $\mathrm{mm}$ slices parallel to the fiber direction, vacuum-packed, and frozen at $-20^{\circ} \mathrm{C}$ until analysis. For sensory evaluation, the beef strips were thawed to approximately $4^{\circ} \mathrm{C}$. Individual strips were cooked by placing them on a tin plate equipped with a water jacket (ca. $245^{\circ} \mathrm{C}$ to $255^{\circ} \mathrm{C}$ ). The strips were turned at the first pooling of liquid on the surface of the sample or at the start of shrinkage. The cooked strips were immediately served to each panelist for evaluation. A total of 7 to 9 trained panelists were used for each session. They scored tenderness, juiciness, and flavor likeness on a 10-point scale from one (very tough; very dry; dislike extremely) to 10 (very tender; very juicy; like extremely). Each sample was tested once independently by each panelist. Scores were averaged over the different panelists for each animal.

\section{Genotype assay}

Genomic DNA for genotyping assays was extracted from the blood samples using DNeasy 96 Blood and Tissue Kit (Qiagen, Valencia, CA, USA). DNA quantification was performed using a NanoDrop 1000 (Thermo Fisher Scientific Inc., Wilmington, DE, USA). DNA samples were submitted for genotyping with total DNA (900 ng), 260/280 ratio >1.8, and DNA concentration $20 \mathrm{ng} / \mu \mathrm{L}$. The genotyping was conducted by the Animal Genome and Bioinformatic Division of National Institute of Animal Science, RDA, Korea using the Bovine 50K BeadChip (Illumine, San Diego, CA, USA; Matukumalli et al., 2009). Stringent filtering criteria were applied to the genotype data. Briefly, SNPs were excluded from the analysis if they failed in $>5 \%$ of the genotypes, had median GC scores $<0.6$, GC scores $<0.6$ in $<90 \%$ of the samples, deviated in heterozygosity $>3$ standard deviations from the other SNPs, 
and were out of Hardy-Weinberg equilibrium for a cut-off $p$ value of $1^{-3}$. SNPs with auto-calling rates $<80 \%$ and a minor allele frequency $<0.001$ were excluded from later analyses. Unmapped SNPs and SNPs on sex chromosomes were also excluded. Individual genotypes with GC scores <0.6 were treated as missing. A total of 38,291 SNPs were included in the analysis.

\section{Statistical analyses}

Phenotype and model definitions: To test the association between the SNP and QTL, a single marker linear mixed model was implemented using ASREML Software. Twentysix markers were assumed to be in LD with QTL in close proximity, and the effect evaluated was the additive effect (QTL allele substitution effect). The additive effect was fitted as a regression of the phenotype on allele counts $(0,1$, and 2). The additive effect of a SNP on each phenotype was calculated by regression analysis, with values in the covariate coded as 0,1 , or 2 copies of the variant allele, and fit to the following mixed model: trait $\sim$ mean+fixed effects $+\mathrm{SNP}_{i}$ +animal+error, with the animal and error fitted as random effects. The $i$-th $\mathrm{SNP}\left(S N P_{i}\right)$ was fitted as a covariate effect. Fixed effects included the year, season, and sex. The day of slaughter was fitted as a covariate effect. Quantile-quantile (Q-Q) plots were investigated to judge the extent of false positive signals. SNPs were considered as significant for $\mathrm{p}$ values below the $5 \%$ Bonferroni-corrected type I error threshold for 38,291 independent statistical tests. Allele substitution effects were estimated for each significant marker in a linear regression model implemented in ASREML.

Proportion of SNP variance: The percentage of the phenotypic variance explained by each significant SNP was calculated. The percentage of the phenotypic variance accounted by the $i$-th SNP was computed according to the following formula:

$$
\% \mathrm{Vp}_{\mathrm{i}}=100 \times \frac{2 p_{i} q_{i} a_{i}^{2}}{\sigma_{\mathrm{p}}^{2}}
$$

Where: $p_{i}$ and $q_{i}$ are the allele frequencies for the $i$-th SNP estimated for Hanwoo, $a_{i}$ is the estimated additive effect of the $i$-th SNP on the phenotype in question, and $\sigma_{\mathrm{p}}^{2}$ is the REML estimate of the phenotypic variance for WB shear force and sensory traits in Hanwoo.

Linkage disequilibrium: Linkage disequilibrium refers to the non-random association of alleles at $2+$ loci and is important in fine-scale mapping of QTL (Meuwissen and Goddard, 2000). To determine if any of the SNPs significantly associated with tenderness are in linkage disequilibrium with the SNPs in the CAST gene, we selected the markers flanking a $1-\mathrm{Mb}$ region of the CAST
SNPs. A total of 24 markers were used to study linkage disequilibrium. Haplotypes were reconstructed using default parameters in PHASE and visualized by means of Heatmap plots obtained with Haploview.

\section{RESULTS AND DISCUSSION}

In this genome-wide association study, 288 cattle were used as a GWAS dataset to identify QTLs for distinct Hanwoo phenotypes of WB-shear force values and sensory traits (tenderness, juiciness, and flavor likeness). The 38,291 SNPs on the autosomes that fulfilled the SNP quality control criteria were used for the association analysis. The GWAS analysis was performed using a linear mixed model approach and a numeric relationship matrix estimated by pedigree between animals. Summary statistics for the data are provided in Table 1. Mean values for tenderness, juiciness, and flavor likeness were slightly higher in the steer group than other 2 sexes, while the WBshear force values were fairly low in the steer group. A total of 18 significant SNPs associated with WB shear force, tenderness, juiciness, and flavor likeness were identified (Table 2). Hapmap40437-BTA-49726 (Chr5; P = 9.29 $\times 10^{-7}$; Position-69686900), ARS-BFGL-NGS-110680 (Chr17; $\mathrm{P}=$ $5.71 \times 10^{-5}$; Position-4423257), Hapmap42498-BTA-29406 (Chr4; P $=1.64 \times 10^{-5}$; Position-19444028), and BTB01204750 (Chr6; P = 1.60×10 $0^{-5}$; Position-13417489) were found to be more significantly associated with WB shear force, tenderness, juiciness, and flavor likeness, respectively. Of the 18 identified SNPs, none had a combined effect on traits analyzed in this study. However, 3 distantly located SNPs on chromosome 7 showed an association with all traits, except meat juiciness. Drinkwater et al. (2005) detected QTL affecting beef tenderness on bovine chromosome 7 near CAST and lysyl oxidase. In this study, with the use of a $50 \mathrm{k}$ SNP chip, significant associations were not detected between the SNPs in the CAST gene and meat tenderness. However, we found a significant SNP (ARS-BFGL-NGS-113774) in the 1-Mb region of the CAST SNPs, but we also observed the absence of the linkage disequilibrium structure (Supplementary Figure S7: See eversion for supplement.).

However, in a separate study, where we specifically genotyped 3 SNPs in the CAST gene and 4 SNPs in the CAPN1 gene, we identified an association between a mutation and meat tenderness, flavor, and juiciness of Hanwoo meat (Lee et al., 2013). Analyzing the high density (HD) SNP chip can be of importance in this context. Casas (2002) identified QTLs for shear force in 4 half-sib resource populations on chromosomes 4, 5, 15, and 29. Keele et al. (1999) identified a region on bovine chromosome 15 that influences beef longissimus tenderness in steers. While we did identify significant associations on chromosomes 4 and 
Table 1. Summary statistics for Warner-Bratzler shear force and sensory evaluation

\begin{tabular}{|c|c|c|c|c|c|c|}
\hline Trait & Sex & $\mathrm{n}$ & Mean & SD & Min & $\operatorname{Max}$ \\
\hline \multirow[t]{4}{*}{ WB shear force } & Cow & 116 & 6.49 & 2.53 & 2.86 & 12.9 \\
\hline & Bull & 90 & 7.21 & 2.61 & 3.07 & 12.8 \\
\hline & Steer & 82 & 3.42 & 0.53 & 2.61 & 5.3 \\
\hline & Total & 288 & 5.85 & 2.68 & 2.61 & 12.9 \\
\hline \multirow[t]{4}{*}{ Tenderness } & Cow & 116 & 3.80 & 0.79 & 1.50 & 5.6 \\
\hline & Bull & 90 & 3.50 & 0.71 & 2.00 & 5.3 \\
\hline & Steer & 82 & 4.45 & 0.51 & 3.20 & 5.3 \\
\hline & Total & 288 & 3.89 & 0.79 & 1.50 & 5.6 \\
\hline \multirow[t]{4}{*}{ Juiciness } & Cow & 115 & 4.04 & 0.76 & 1.70 & 5.3 \\
\hline & Bull & 90 & 4.16 & 0.51 & 2.80 & 5.4 \\
\hline & Steer & 82 & 4.68 & 0.39 & 3.70 & 5.7 \\
\hline & Total & 287 & 4.26 & 0.65 & 1.70 & 5.7 \\
\hline \multirow[t]{4}{*}{ Flavor-likeness } & Cow & 116 & 4.24 & 0.47 & 3.00 & 5.5 \\
\hline & Bull & 90 & 4.25 & 0.42 & 3.00 & 5.3 \\
\hline & Steer & 82 & 4.62 & 0.35 & 3.60 & 5.5 \\
\hline & Total & 288 & 43.50 & 0.46 & 3.00 & 5.5 \\
\hline
\end{tabular}

SD, standard deviation; WB, Warner-Bratzle.

5, no association was detected on chromosomes 15 and 29. 21 (Position: 34318948 Intron1) with juiciness. We Dunner et al. (2013) identified an association on identified associations of SNP ARS-BFGL-NGS-80140 on chromosome 4 (Position: 114895056 Exon 7-S, 114894952 chromosome 4 and ARS-BFGL-NGS-119377 on Intron 7) with m-calpain and an association on chromosome chromosome 21 in the region close to that identified by

Table 2. SNPs showing significant association with Warner-Bratzler shear force and sensory evaluation in 288 Hanwoo animals

\begin{tabular}{|c|c|c|c|c|c|c|c|c|c|c|c|}
\hline$\overline{\mathrm{SNP}}$ & $\mathrm{Chr}$ & Position & $\mathrm{p}$ value & $\mathrm{n}^{a}$ & $\mathrm{n} 0^{b}$ & $\mathrm{n} 1^{c}$ & $\mathrm{n} 2^{d}$ & $\overline{\mathrm{P}^{e}}$ & $\mathrm{Q}^{f}$ & $\mathrm{Vp}$ & $\beta$ \\
\hline \multicolumn{12}{|l|}{ WB shear force } \\
\hline Hapmap40437-BTA-49726 & 5 & 69686900 & $9.29 \times 10^{-7}$ & 283 & 35 & 140 & 108 & 0.37 & 0.63 & 0.12 & 0.82 \\
\hline ARS-BFGL-NGS-114241 & 11 & 100472048 & $2.56 \times 10^{-5}$ & 287 & 31 & 135 & 121 & 0.34 & 0.66 & 0.09 & -0.72 \\
\hline ARS-BFGL-NGS-104667 & 11 & 99888515 & $3.33 \times 10^{-5}$ & 287 & 82 & 135 & 70 & 0.52 & 0.48 & 0.08 & 0.63 \\
\hline BTB-01632466 & 7 & 2346546 & $8.52 \times 10^{-5}$ & 283 & 163 & 105 & 15 & 0.76 & 0.24 & 0.09 & 0.78 \\
\hline \multicolumn{12}{|l|}{ Tenderness } \\
\hline ARS-BFGL-NGS-110680 & 17 & 4423257 & $5.71 \times 10^{-5}$ & 284 & 137 & 129 & 18 & 0.71 & 0.29 & 0.09 & 0.28 \\
\hline BTB-00299070 & 7 & 25511561 & $6.20 \times 10^{-5}$ & 287 & 2 & 34 & 251 & 0.07 & 0.93 & 0.09 & 0.50 \\
\hline \multicolumn{12}{|l|}{ Juiciness } \\
\hline Hapmap42498-BTA-29406 & 4 & 19444028 & $1.64 \times 10^{-5}$ & 287 & 225 & 59 & 3 & 0.89 & 0.11 & 0.10 & -0.35 \\
\hline Hapmap47860-BTA-120557 & 21 & 9600154 & $2.33 \times 10^{-5}$ & 288 & 118 & 133 & 37 & 0.64 & 0.36 & 0.10 & -0.23 \\
\hline ARS-BFGL-NGS-119377 & 21 & 37931511 & $4.69 \times 10^{-5}$ & 286 & 217 & 61 & 8 & 0.87 & 0.13 & 0.07 & -0.28 \\
\hline BTB-01582726 & 8 & 4304423 & $5.20 \times 10^{-5}$ & 286 & 245 & 39 & 2 & 0.92 & 0.08 & 0.08 & -0.39 \\
\hline BTA-86030-no-rs & 8 & 4340819 & $5.20 \times 10^{-5}$ & 285 & 245 & 38 & 2 & 0.93 & 0.07 & 0.08 & -0.39 \\
\hline ВТВ-01374425 & 9 & 235048 & $7.19 \times 10^{-5}$ & 286 & 3 & 72 & 211 & 0.14 & 0.86 & 0.09 & 0.31 \\
\hline \multicolumn{12}{|l|}{ Flavor likeness } \\
\hline ВТВ-01204750 & 6 & 13417489 & $1.60 \times 10^{-5}$ & 283 & 114 & 123 & 46 & 0.62 & 0.38 & 0.10 & 0.17 \\
\hline ARS-BFGL-NGS-29419 & 25 & 39112932 & $1.99 \times 10^{-5}$ & 286 & 21 & 107 & 158 & 0.26 & 0.74 & 0.10 & 0.19 \\
\hline ARS-BFGL-NGS-105712 & 28 & 27455951 & $3.20 \times 10^{-5}$ & 288 & 158 & 119 & 11 & 0.76 & 0.24 & 0.09 & 0.19 \\
\hline ARS-BFGL-NGS-113487 & 23 & 49155568 & $4.79 \times 10^{-5}$ & 288 & 208 & 74 & 6 & 0.85 & 0.15 & 0.09 & -0.22 \\
\hline BTB-01207822 & 7 & 43132401 & $5.84 \times 10^{-5}$ & 284 & 65 & 148 & 71 & 0.49 & 0.51 & 0.09 & 0.16 \\
\hline ARS-BFGL-NGS-80140 & 4 & 113555764 & $6.46 \times 10^{-5}$ & 281 & 176 & 101 & 4 & 0.81 & 0.19 & 0.10 & 0.21 \\
\hline
\end{tabular}

SNP, single nucleotide polymorphism; WB, Warner-Bratzler.

The allelic substitution effect $(\beta)$ is given for the minor allele in the additive genetic effect derived from a linear regression model of WB shear force and sensory traits. The Vp indicates the proportion of SNP vairance calculated from the equation in the Material and Methods.

${ }^{a}$ Number of samples with total non-missing genotypes. ${ }^{b}$ Number of samples with 0 genotypes.

${ }^{c}$ Number of samples with 1 genotype. ${ }^{d}$ Number of samples with 2 genotypes. ${ }^{e, f}$ Allele frequency. 
Dunner et al. (2013).

In addition, the allele substitution effect for each of the significant SNPs was calculated from linear regression analysis. The most significant SNPs (Hapmap40437-BTA49726; P = 9.29 $\times 10^{-7}$, Hapmap42498-BTA-29406; P = 1.64 $\times 10^{-5}$, ARS-BFGL-NGS-110680; $\mathrm{P}=5.71 \times 10^{-5}$, BTB$01204750 ; \mathrm{P}=1.60 \times 10^{-5}$ ) had $0.82,-0.35,0.28$, and 0.17 allele substitution effects for WB shear force, tenderness, juiciness, and flavor likeness, respectively (Table 2).

The Q-Q plot with $\mathrm{p}<10^{-3}$ for WB shear force and sensory evaluation revealed only minimal deviation from the distribution under the null hypothesis (Figure 1; Supplementary Figures S1, S2, and S3: See e-version for supplement.). We calculated the proportion of phenotypic variance explained by the SNPs with a $\mathrm{p}$ value of 0.001 (Figure 2; Supplementary Figures S4, S5, and S6: See eversion for supplement.). As shown in Figure 2, each of the SNPs between a threshold of $\mathrm{p}=0.001$ and $\mathrm{p}=0.0001$ explained $3 \%$ to $9 \%$ of the phenotypic variance, which is only a small proportion when compared to the total phenotypic variance. However, the most significant SNPs accounted for $7 \%$ to $12 \%$ of phenotypic variance, which is a large proportion when compared to the total phenotypic variance.
A number of SNPs showed slight significant $(\mathrm{p}<0.001)$ associations with WB shear force over tenderness, juiciness, or flavor likeness. There were 50, 25, 29, and 34 SNPs with significant associations with WB shear force, tenderness, juiciness, and flavor likeness, respectively. Bolormaa et al. (2011) reported GWAS for meat tenderness measured as peak force to shear (LLPF) to identify 64 significant SNPs $(\mathrm{p}<0.001)$ in 940 Australian beef cattle, and there were 5 chromosomes (i.e., 7, 8, 12, 22, and 29) comprising the largest number of significant SNPs at $\mathrm{p}<0.001$. In this study, there were WB shear force $(1,7,11,14,23$, and 25), tenderness $(7,23$, and 25), juiciness $(8,14$, and 21), and flavor likeness (4, 5, and 6) chromosomes with >3 significant SNPs. Despite the differences in breed (i.e., Hanwoo vs Australian beef cattle), 3 traits (LLPFAustralian beef cattle, WB shear force, and tendernessHanwoo) measuring meat tenderness were shown to possess the same major chromosome (i.e., BTA7). Moreover, WB shear force and tenderness in Hanwoo had associations with major chromosomes 23, 25, and 7. These may be the most important chromosomes (i.e., BTA7, 23, and 25) associated meat tenderness in Hanwoo.

The previous sensory test results showed that Korean consumers decided the palatability of Hanwoo beef by
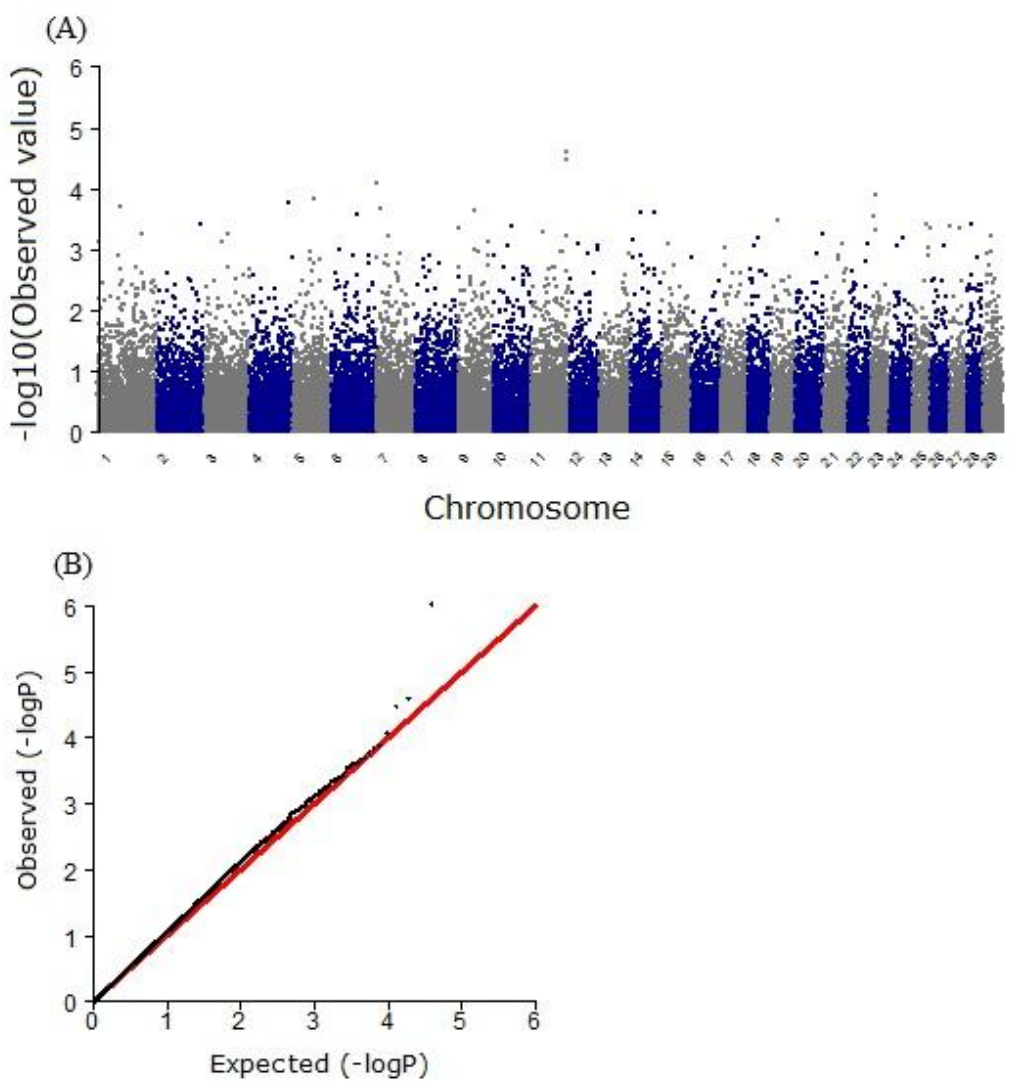

Figure 1. Association of 38,291 SNPs with the WB shear force. (A) Manhattan plot. (B) Quantile-quantile plot. The red line represents the $95 \%$ concentration band under the null hypothesis of no association. The black dot represents the p values of the entire study. SNP, single nucleotide polymorphism; WB, Warner-Bratzler. 
Vp for WB-Shear force

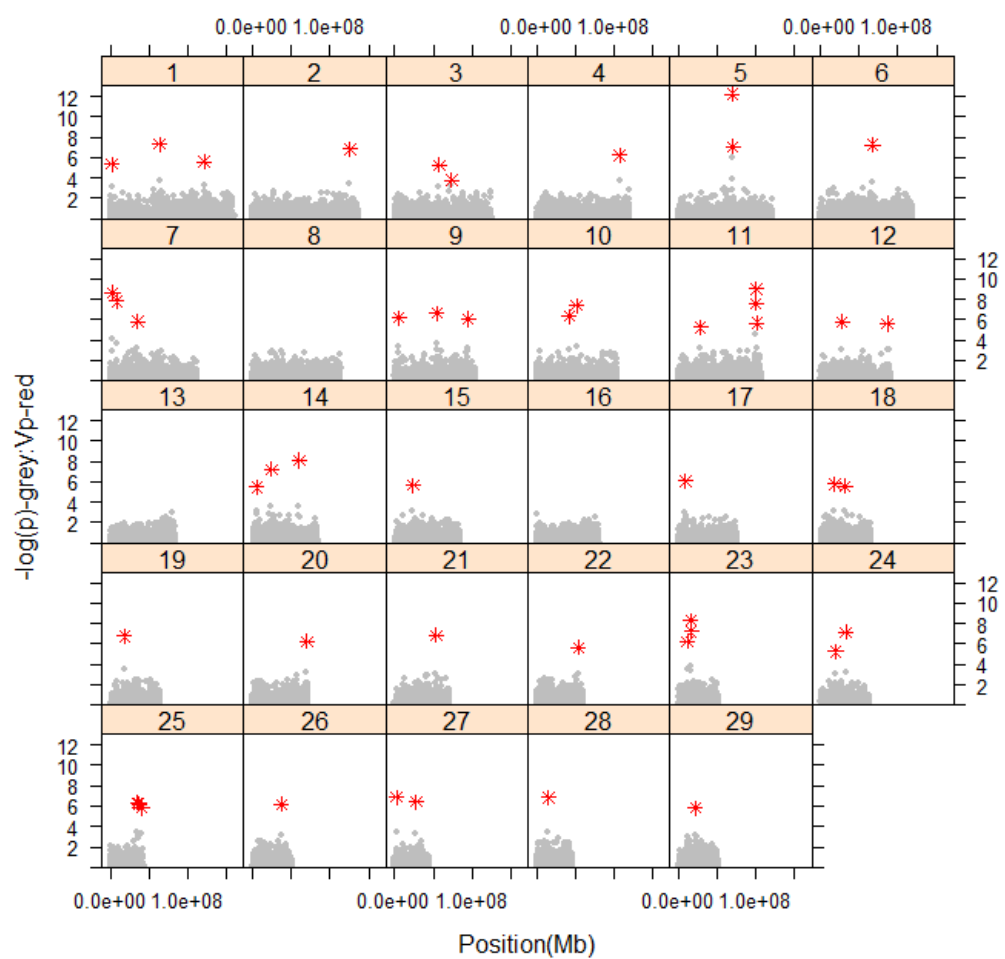

Figure 2. The - $\log p$ value for the association between SNP and WB shear force (grey) and phenotypic variance (red) that significant SNPs $(p<0.001)$ account for in the single marker regression analysis. SNP, single nucleotide polymorphism; WB, Warner-Bratzler.

judging tenderness (55\%), juiciness (18\%), and flavor likeness (27\%) (Cho et al., 2010). Tenderness is considered the most important qualitative characteristic of meat, and the consumer is willing to pay a higher price in the market place for beef as long as it is guaranteed to be tender (Miller et al., 2001). Tenderness can be evaluated by instrumental tests, such as WB-shear force and sensorial panel tests. The correlation coefficient of WB-shear force measurements with tenderness sensory ratings was -0.72 (Destefanis et al., 2008).

In this study, for WB shear force and sensory traits (tenderness, juiciness, and flavor likeness), only one SNP was found to meet the Bonferroni-corrected threshold $\left(\mathrm{p}<1.3 \times 10^{-6}\right)$, and the phenotypic variance appeared to be an overestimation in proportion to the significant threshold. These results might be due to the inability of the sample size $(n=288)$ to successfully map QTL with a small proportion of genetic variation for a complex trait. To achieve an accurate estimation of the SNP effects on traits of interest in GWAS, larger population size and higher density of the markers are required. Pausch et al. (2012) calculated the adequate sample size required to successfully map QTL for a complex trait with very low heritability. He assumed a heritability of 0.08 , a linkage disequilibrium between marker SNPs and QTL of $r^{2}=0.35$, and a QTL explanation of $4 \%$ of the genetic variation, which would require 20,000 animals for the successful identification of QTL.

In the dearth of studies considering GWAS on WB shear force and sensory panel traits, this study can act as a primer. The results of this study will be useful in providing fundamental information for genetic improvement in the Hanwoo breed to increase consumer reliability through the implementation of molecular information for palatable quality management. However, because WB shear force and sensory evaluation are affected by a few or many loci with moderate or minimal effects, respectively, across the genome, further study will be needed to examine the WB shear force and sensory traits utilizing a larger population size and HD SNP chip in Hanwoo.

\section{ACKNOWLEDGMENTS}

This study was supported by awards from the AGENDA project (Grant no. PJ010170) and Molecular Breeding Program of Next Generation BIOGREEN 21 project (Grant no. PJ009954) in the National Institute of Animal Science, Rural Development Administration, Korea.

\section{REFERENCES}

Bolormaa, S., L. R. Porto Neto, Y. D. Zhang, R. J. Bunch, B. E. 
Harrison, M. E. Goddard, and W. Barendse. 2011. A genomewide association study of meat and carcass traits in Australian cattle. J. Anim. Sci. 89:2297-2309.

Caine, W. R., J. L. Aalhus, D. R. Best, M. E. R. Dugan, and L. E. Jeremiah. 2003. Relationship of texture profiles analysis and Warner-Bratzler shear force with sensory characteristics of beef rib steaks. Meat Sci. 64:333-339.

Carmack, C. F., C. L. Kastner, M. E. Dikeman, J. R. Schwenke, and C. M. Garcia Zepeda. 1995. Sensory evaluation of beef flavor intensity, tenderness and juiciness among major muscles. Meat Sci. 39:143-147.

Casas, E. 2002. Identification of quantitative trait loci in beef cattle. Archivos Latinoamericanos de Produccion Anim. 10: 54-61.

Casas, E., S. N. White, D. G. Riley, T. P. L. Smith, R. A. Brenneman, T. A. Olson, D. D. Johnson, S. W. Coleman, G. L. Bennett, and C. C. Chase. 2005. Assessment of single nucleotide polymorphisms in genes residing on chromosomes 14 and 29 for association with carcass composition traits in Bos indicus cattle. J. Anim. Sci. 83:13-19.

Casas, E., S. N. White, T. L. Wheeler, S. D. Shackelford, M. Koohmaraie, D. G. Riley, C. C. Chase, D. D. Johnson, and T. P. L. Smith. 2006. Effects of calpastatin and (micro)-calpain markers in beef cattle on tenderness traits. J. Anim. Sci. 84: 520-525.

Cho, S. H., J. Kim, B. Y. Park, P. N. Seong, G. H. Kim, S. G. Jung, S. K. Im, and D. H. Kim. 2010. Assessment of meat quality properties and development of a palatability prediction model for Korean Hanwoo steer beef. Meat Sci. 86:236-242.

Destefanis, G., A. Brugiapaglia, M. T. Barge, and E. Dal Molin. 2008. Relationship between beef consumer tenderness perception and Warner-Bratzler shear force. Meat Sci. 78:153156.

Drinkwater, R. D., Y. Li, I. Lenane, G. P. Davis, R. Shorthose, B. E. Harrison, K. Richardson, D. Ferguson, R. Stevenson, J. Renaud, I. Loxton, R. J. Hawken, M. B. Thomas, S. Newman, D. J. S. Hetzel, and W. Barendse. 2006. Detecting quantitative trait loci affecting beef tenderness on bovine chromosome 7 near calpastatin and lysyl oxidase. Aust. J. Exp. Agric. 46:159164.

Dunner, S., N. Sevane, D. García, O. Cortés, A. Valentini, J. L. Williams, B. Mangin, J. Cañón, and H. Levéziel. 2013. The GeMQual consortium. Association of genes involved in carcass and meat quality traits in 15 European bovine breeds. Livest. Sci. 154:34-44.

Jeremiah, L. E. 1982. Consumer preferences regarding cooking methods and times for beef loin steaks in central Alberta. J. Consum. Stud. Home Econ. 6:79-86.

Keele, J. W., S. D. Shackelford, S. M. Kappes, M. Koohmaraie, and R. T. Stone. 1999. A region on bovine chromosome 15 influences beef longissimus tenderness in steers. J. Anim. Sci. 77:1364-1371.

Koohmaraie, M. 1994. Muscle proteinases and meat ageing. Meat Sci. 36:93-104

Koohmaraie, M., M. P. Kent, S. D. Shackleford, E. Veiseth, and T. L. Wheeler. 2002. Meat tenderness and muscle growth: Is there any relationship. Meat Sci. 62:345-352.

Lee, S. H., S. C. Kim, H. H. Chai, S. H. Cho, D. J. Lim, B. H.
Choi, C. G. Dang, C. Gondro, B. S. Yang, and S. K. Hong. 2013. Mutations in calpastatin and $\mu$-calpain are associated with meat tenderness, flavor, and juiciness of Hanwoo (Korean cattle): Molecular modeling of the effects of substitutions in the calpastatin/ $\mu$-calpain complex. Meat Sci. 96:1501-1508.

Love, J. 1994. Product acceptability evaluation. In: Quality Attributes and Their Measurement in Meat, Poultry and Fish Products. (Eds. A. M. Pearson and T. R. Dutson). Blackie Academic and Professional, Glasgow, UK. Adv. Meat Res. 9:337-358

Marshall, D. M. 1999. Genetics of Meat Quality. CABI International, Oxfordshire, UK.

Matukumalli, L. K., C. T. Lawley, R. D. Schnabel, J. F. Taylor, M. F. Allan, M. P. Heaton, J. O'Connell, S. S. Moore, T. P. L. Smith, T. S. Sonstegard, and C. P. VanTassell. 2009. Development and characterization of a high density SNP genotyping assay for cattle. Plos one. 4(4):e5350.

Meuwissen, T. H. E. and M. E. Goddard. 2000. Fine mapping quantitative trait loci using linkage disequilibria with closely linked marker loci. Genetics 155:421-430.

Miller, M. F., M. A. Carr, C. B. Ramsey, K. L. Crokett, and L. C. Hoover. 2001. Consumer thresholds for establishing the values of beef tenderness. J. Anim. Sci. 79:3062-3068.

Page, B. T., E. Casas, M. P. Heaton, N. G. Cullen, D. L. Hyndman, C. A. Morris, A. M. Crawford, T. L. Wheeler, M. Koohmaraie, J. W. Keele, and T. P. L. Smith. 2002. Evaluation of singlenucleotide polymorphisms in CAPN1 for association with meat tenderness in cattle. J. Anim. Sci. 80:3077-3085.

Page, B. T., E. Casas, R. L. Quaas, R. M. Thallman, T. L. Wheeler, S. D. Shackelford, M. Koohmaraie, S. N. White, G. L. Bennett, J. W. Keele, M. E. Dikeman, and T. P. L. Smith. 2004. Association of markers in the bovine CAPN1 gene with meat tenderness in large crossbred populations that sample influential industry sires. J. Anim. Sci. 82:3474-3481.

Pausch, H., K. Flisikowski, S. Jung, R. Emmerling, C. Edel, K. Gotz, and R. Fries. 2011. Genome-wide association study identifies two major loci affecting calving ease and growthrelated traits in cattle. Genetics 187:289-297.

Rolf, M. M., S. D. Mckay, M. C. McClure, J. E. Decker, T. M. Taxis, R. H. Chapple, D. A. Vasco, S. J. Gregg, J. W. Kim, R. D. Schnabel, and J. F. Taylor. 2010. How the next generation of genetic technologies will impact beef cattle selection. Proceedings of the Beef Improvement Federations 42nd Annual Research Symposium and Annual Meeting, Columbia, MO, USA. 46-56.

Schenkel, F. S., S. P. Miller, Z. Jiang, I. B. Mandell, X. Ye, H. Li, and J. W. Wilton. 2006. Association of a single nucleotide polymorphism in the calpastatin gene with carcass and meat quality traits of beef cattle. J. Anim. Sci. 84:291-299.

Shackelford, S. D., T. L. Wheeler, M. K. Meade, J. O. Reagan, B. L. Byrnes and M. Koohmaraie. 2001. Consumer impressions of Tender Select beef. J. Anim. Sci. 79:2605-2614.

Shackelford, S. D., T. L. Wheeler, and M. Koohmaraie. 2005. Online classification of US Select beef carcasses for longissimus tenderness using visible and near-infrared reflectance spectroscopy. Meat Sci. 69:409-415.

Shahidi, F. and L. J. Rubin. 1986. Meat flavor volatiles: A review of the composition, techniques of analysis, and sensory 
evaluations. CRC Crit. Rev. Food Sci. Nutr. 24:141-243.

Wheeler, T. L., S. D. Shackelford, and M. Koohmaraie. 2000. Relationship of beef longissimus tenderness classes to tenderness of gluteus medius, semimembranosus, and biceps femoris. J. Anim. Sci. 78:2856-2861.
White, S. N., E. Casas, T. L. Wheeler, S. D. Shackelford, M. Koohmaraie, D. G. Riley, C. C. Chase, Jr., D. D. Johnson, J. W. Keele, and T. P. L. Smith. 2005. A new single nucleotide polymorphism in CAPN1 extends the current tenderness marker test to include cattle of Bos indicus, Bos taurus, and crossbred descent. J. Anim. Sci. 83:2001-2008. 